\title{
A Secure and Efficient Authentication Scheme for Personal Health Care System
}

\author{
Chin-Ling Chen ${ }^{1,2, a}$, Po-Tsun Huang ${ }^{1, b}$ and Yong-Yuan Deng ${ }^{1, c}$, \\ ${ }^{1}$ Department of Computer Science and Information Engineering, Chaoyang University of Technology, 168 Jifeng E. Rd., Wufeng \\ District, Taichung, 41349 Taiwan, R.O.C. \\ ${ }^{2}$ School of Information Engineering, Changchun Sci-Tech University, Changchun 130600, P.R. China \\ aclc@mail.cyut.edu.tw, ${ }^{b} \mathrm{~m} 4596646 @$ cycu.org.tw, ${ }^{\mathrm{c} a l l e n . n u b i @ g m a i l . c o m, ~}$
}

\begin{abstract}
With the development of network environment and hardware technology, smart devices therefore have become very popular tools. Nowadays, smart facilities are very common in our daily lives. For example, smart refrigerators, smart air conditioners, smart home, smart cities and a large number of applications of Internet of Things have been used. The concept and technology of Internet of Things are being carried out in full swing. Internet of Things in the personal care is one of the concern topics. In order to prevent malicious attacks, resulting in the leakage of patient data, the security of Internet of Things is important. Review the related works about health care service. We found that their article lack of complete security mechanism, therefore we proposed a new architecture which is an IoT-based authentication scheme for personal health care system. In the proposed scheme, doctors can get patients' health status from wearable devices of patients. We complete the goal of electronic medical record sharing. Our scheme can achieve anonymity, mutual authentication, non-repudiation. It can also against forgery attack.
\end{abstract}

Key words: Internet of Things, health care, electronic medical record, forgery attack

\section{Introduction}

In view of the ageing population and the decreasing birth rate, there are many people live alone especially the elders. According to the statistic publish by WHO in 2015 [1]. We found that it is important to face the increasing rate of sudden death. In order to adapt the current society and avoid by sudden death, wearable device in wireless sensor network would be helpful [2]. It makes sense in detecting the patients' symptoms. Wearable device sent out a warning before the symptom getting worse [3]. It may decrease the probability of sudden death.

In general, the wearable devices are functional in IoT, but the resources in IoT are constrained [4]. It is said that the wearable devices restrict in low computation. We avoid high computational encryption method in our scheme when we deliver the message, so it is important of using simple way to reach the security level [5]. Moreover, the wearable devices are used in wireless, it may cause attacks by the adversary [6]. These security issues include privacy and authentication are the most important things must be considered [7]. Therefore, we proposed a secure IoT structure to solve these problems. Our scheme achieved the follow factors: (1) anonymity, (2) mutual authentication, (3) non-repudiation, (4) avoid forgery attack [8].

The rest of the paper organized as follows: we detailed our

proposed scheme in session 2 . Next, we analyzed the security in session 3. Finally, we made a conclusion of our paper in session 4 .

\section{The Proposed Scheme}

\section{A. System architecture}

In this paper, we use the public-key cryptography to transmit the session keys [9]. Consequently, we used the symmetric-key cryptography to transmit the diagnosed records. The architecture of our scheme is shown in Figure 1.
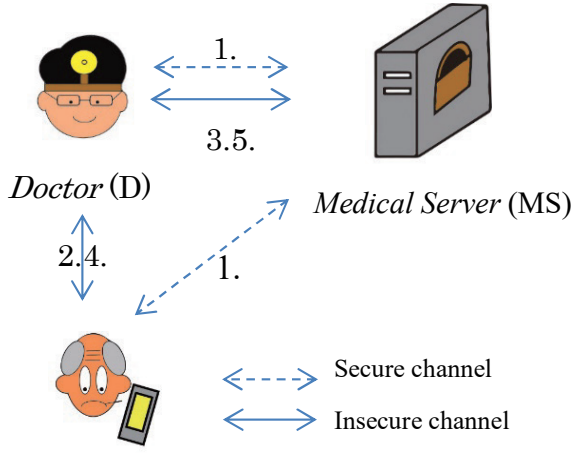

\section{Patient (P)}

Fig. 1. The architecture of personal health care system

There are three parties involve the personal health system.

1. Patient: the owners of the medical mobile device. In anytime, the patient can send the health information to the doctor via the mobile device.

2. Doctor: the one diagnoses the information sent by the patient and stored the data in the medical server.

3. Medical server: the equipment stores the health record diagnosed by the doctor.

Our scheme divided into two phases. First, the registration phase that the patient and the doctor should register to the medical server. The patient and the doctor saved their IDs in the medical server. Second, the patient will go to see a doctor. This phase is called the doctor visiting phase. The doctor will receive the health status from the patient's mobile device. Then, the doctor will show his doctor license to get the diagnostic record. Therefore, the doctor can make a diagnosis. At the end, the doctor returns the diagnosed information to the patient. Also updates the electrical medical record to the medical server. The steps are described as below:

Step 1: At the beginning, the patient and the doctor register to medical server.

Step 2: Then the patient goes to see a doctor for a medical diagnose in person. 
Step 3: The doctor receives the health record which stores in the medical server.

Step 4: The doctor then makes a professional diagnosed the information from the patient and the medical server.

Step 5: The doctor sends the diagnostic information to the patient and updates the electrical medical record in the medical server.

\section{B. Notations}

The notations of the proposed scheme are shown in table 1.

Table 1. Notations of this scheme

\begin{tabular}{ll}
\hline Notations & \multicolumn{1}{c}{ Means } \\
\hline$I D_{x}$ & $:$ identity of $x$ \\
$S K_{x}$ & $:$ session key of $x$ \\
$r_{x}$ & $:$ random numbers are generated by $x$ \\
Cert $D$ & $:$ the doctor's certificate \\
$h() \quad:$. one-way hash function \\
$\|$ & $:$ concatenation function \\
$A_{=}^{?} B \quad$ : determines if $A$ is equal to $B$ \\
$--->\quad$ : secure channel \\
$->\quad$ insecure channel
\end{tabular}

\section{The registration phase}

In this phase, the patient chooses a doctor to visit. The patient shares the identity and the information to the doctor. And then the doctor registers to the medical server, the key generation center generates unique information. Both the doctor and the patient store the unique information generates by the medical server, the details are shown as follows:

Step 1: The patient chooses a random number $r_{P}$. The patient uses his/her identity $I D_{P}$ and the random number $r_{P}$ to compute a masked identity $M I D_{P}$ as follows:

$M I D_{P}=h\left(I D_{P} \| r_{P}\right)$.

Then, the patient transmits the identity and the masked identity to the doctor through the secure channel.

Step 2: After receiving the message, the doctor generates a random number $r_{D}$. And the doctor combines his/her identity and his/her masked identity to computes the masked identity $M I D_{D}$ as follows:

$M I D_{D}=h\left(I D_{D} \| r_{D}\right)$.

The doctor stores $M I D_{P}$ and $I D_{P}$. And the doctor sends the patient's identity $I D_{p}$, the masked identity $M I D_{P}$ of patient, the doctor's identity $I D_{D}$, the masked identity $M I D_{D}$ of doctor and the doctor's license Cert $_{D}$ to the medical server.

Step 3: Upon receiving the messages, the medical server generates a random number $r_{P D}$. And uses the random number $r_{P D}$, the patient's masked identity $M I D_{P}$ and the masked identity $M I D_{D}$ of doctor to compute the masked identity $M I D_{M S}$ as follows:

$M I D_{M S}=h\left(r_{P D}\left\|M I D_{P}\right\| M I D_{D}\right)$.

And the medical server stores the patient's identity $I D_{P}$, the patient's masked identity $M I D_{P}$, the doctor's identity $I D_{D}$ of doctor, the masked identity $M I D_{D}$ of doctor. And then the medical server sends the message $M I D_{M S}$ to the doctor.

Step 4: After receiving the message, the doctor stores the masked identity $M I D_{M S}$ of medical server.
Step 5: Then, the doctor sends the identity $I D_{D}$ of doctor, the masked identity $M I D_{D}$ of doctor and masked identity $M I D_{M S}$ of the medical server to the patient.

Step 6: After receiving the message, the patient stores the doctor's identity $I D_{D}$, the masked identity $M I D_{D}$ of doctor and the masked identity $M I D_{M S}$ of the medical server.

\section{The doctor diagnosed phase}

In this phase, the doctor can get the health status from the patient. The doctor also can find the diagnosed record from the medical server. When the doctor completely diagnosed the patient. He/she will send the diagnosis to the medical server and the patient. The details are as follows:

Step 1: The patient uses the doctor's public key $P U K_{D}$ to encrypt his/her identity $I D_{P}$, the doctor's identity $I D_{D}$, and the health status $M_{I N F}$. And the patient also signs with his/her identity $I D_{P}$ and the patient's masked identity $M I D_{P}$ as follows:

$C_{P}=E_{P U K D}\left(I D_{P}\left\|I D_{D}\right\| M_{I N F}\right)$.

$\operatorname{Sig}_{P}=S_{P R K}\left(M I D_{P} \| I D_{P}\right)$.

And then, the patient sends $C_{p}$ and $\operatorname{Sig}_{P}$ to the doctor through an insecure channel.

Step 2: After receiving the message, the doctor decrypts the message $C_{p}$ as follows:

$I D_{P}\left\|I D_{D}\right\| M_{I N F}=D_{P P K D}\left(C_{P}\right)$.

The doctor checks if the signature $\operatorname{Sig}_{P}$ is valid or not as follows:

$M I D_{P} \| I D_{P} \stackrel{?}{=} V_{P U K D}\left(\operatorname{Sig}_{P}\right)$.

If it is not valid, the doctor will terminate the diagnosed phase.

Step 3: The doctor uses the medical server's public key $P U K_{M C}$ to encrypt the patient's identity $I D_{P}$, the doctor's identity $I D_{D}$ and the doctor's certificate $\operatorname{Cert}_{D}$. And the doctor also signs with his/her identity $I D_{D}$, the masked identity $M I D_{D}$ of doctor and the doctor's certificate as follows:

$C_{D}=E_{\text {PUKMS }}\left(I D_{P}\left\|I D_{D}\right\| \operatorname{Cert}_{D}\right)$.

$\operatorname{Sig}_{D}=S_{P R K D}\left(I D_{D}\left\|M I D_{D}\right\| \operatorname{Cert}_{D}\right)$.

And then, the doctor sends $M I D_{D}, C_{D}$, and $\operatorname{Sig}_{D}$ to the medical server through an insecure channel.

Step 4: After receiving the message, the medical server decrypts the message $C_{D}$ as follows:

$I D_{P}\left\|I D_{D}\right\| \operatorname{Cert}_{D}=D_{\text {PRKMS }}\left(C_{D}\right)$.

The medical server checks if the signature $\operatorname{Sig}_{D}$ is valid or not as follows:

$I D_{D}\left\|M I D_{D}\right\| \operatorname{Cert}_{D} \stackrel{?}{=} V_{P U K D}\left(\operatorname{Sig}_{D}\right)$.

If it is not valid, the medical server will terminate the diagnosed phase.

Step 5: The medical server uses the doctor's identity $I D_{D}$, the doctor's masked identity $M I D_{D}$, the medical server's identity $I D_{M S}$ and the medical masked identity $M I D_{M S}$ of server to compute the session key $S K_{M S}$ as follows: $S K_{M S}=h\left(I D_{D}\left\|I D_{M S}\right\| M I D_{D} \| M I D_{M S}\right)$.

Afterward, the medical server uses the doctor's public key $P U K_{D}$ to encrypt the session key $S K_{M S}$, the patient's identity $I D_{P}$, the doctor's identity $I D_{D}$ and the medical server's identity $I D_{M S}$. Also signs with the medical server's identity $I D_{M S}$, the medical server's masked identity $M I D_{M S}$ and the session key $S K_{M S}$ as 
follows:

$C_{M S}=E_{P U K D}\left(S K_{M S}\left\|I D_{P}\right\| I D_{D} \| I D_{M S}\right)$.

$\operatorname{Sig}_{M S}=S_{P R K M S}\left(I D_{M S}\left\|M I D_{M S}\right\| S K_{M S}\right)$.

And then, the medical server uses the session key $S K_{M S}$ to encrypt the electrical medical record $M_{E M R}$ as follows:

$C_{S K M S}=E_{S K M S}\left(M_{E M R}\right)$.

The medical server sends $C_{M S}, S_{i g}$ and $C_{S K M S}$ to the doctor.

Step 6: After receiving the message, the doctor decrypts the message $C_{M S}$ as follows:

$S K_{M S}\left\|I D_{P}\right\| I D_{D} \| I D_{M S}=D_{P R K D}\left(C_{M S}\right)$.

The doctor checks if the signature $\mathrm{Sig}_{M S}$ is valid or not $I D_{M S}\left\|M I D_{M S}\right\| S K_{M S} \stackrel{?}{=} V_{P U K M S}\left(\operatorname{Sig}_{M S}\right)$.

If it is valid, the doctor will decrypt the message $M_{E M R}$ as follows:

$M_{E M R}=D_{S K M S}\left(C_{S K M S}\right)$.

Step 7: The doctor uses the patient's public key $P U K_{P}$ to encrypt the patient's identity $I D_{P}$, the doctor's identity $I D_{D}$ and the diagnostic information $M_{D I N F}$ which is diagnosed by the doctor. And the doctor also signs with his/her identity $I D_{D}$, the doctor's masked identity $M I D_{D}$ and the diagnostic information $M_{D I N F}$ as follows:

$C_{D 2}=E_{P U K P}\left(I D_{P}\left\|I D_{D}\right\| M_{D I N F}\right)$.

$\operatorname{Sig}_{D 2}=S_{P R K D}\left(I D_{D}\left\|M I D_{D}\right\| M_{D I N F}\right)$.

The doctor uses the patient's public key $P U B_{P}$ to encrypt the doctor's identity $I D_{D}$, the patient's identity $I D_{P}$, new electrical medical record $M_{E M R}^{N E W}$ which is diagnosed by the doctor and the doctor's certificate Cert $_{D}$ as follows:

$C_{D 3}=E_{S K M S}\left(I D_{D}\left\|I D_{P}\right\| M_{E M R}^{N E W} \| \operatorname{CertD}\right)$.

The doctor sends $C_{D 2}$ and $S_{i g}$ to the patient and sends $C_{D 3}$ and $M I D_{D}$ to the medical server.

Step 8: After receiving the message, the patient decrypts the message $C_{D 2}$ as follows:

$I D_{P}\left\|I D_{D}\right\| M_{D I N F}=D_{P R K P}\left(C_{D 2}\right)$.

The patient checks if the signature $\mathrm{Sig}_{D 2}$ is valid or not as follows:

$I D_{D}\left\|M I D_{D}\right\| M_{D I N F} \stackrel{?}{=} V_{P U K D}\left(\operatorname{Sig}_{D 2}\right)$.

Step 9: Once receiving the message, the medical server uses the session key $S K_{M S}$ to decrypt the message $C_{D 3}$ as follows:

$I D_{P}\left\|I D_{D}\right\| M_{E M R}^{N E W} \| \operatorname{Cert} D=D_{S K M S}\left(C_{D 3}\right)$.

If the certificate $\operatorname{Cert}_{D}$ is valid, the medical server updates the electrical medical record as follows:

$M_{E M R}=M_{E M R}^{N E W}$.

\section{Security Analysis}

\section{A. Anonymity}

In our scheme, all IDs of the parties are encrypted by the following Eqs. (4), (8), (13), (19), (21) during the authentication. So, our scheme achieves anonymity.

Table 2. The non-repudiation proof of our scheme

\section{B. Mutual authentication}

Mutual authentication means the parties that authenticates each other during communication [10]. The authenticate phases was divided into two parts.

(1) Authenticate between the patient and the doctor:

In our scheme, the patient authenticates the doctor server through verifying $\mathrm{Sig}_{D 2}$ as Eq. (23). And the doctor authenticates the patient through verifying $\operatorname{Sig}_{P}$ as Eq. (7).

(2) Authenticate between the doctor and the medical server:

In our scheme, the doctor authenticates the medical server through verifying Sig $_{M S}$ as following Eq. (17). Also the medical server authenticates the patient through verifying $\operatorname{Sig}_{D}$ as Eq. (11).

Therefore, our scheme achieves mutual authentication.

\section{Forgery attack}

In our scheme, the patient makes a signature during the authentication phase as following Eq. (5). The doctor makes a signature during the authentication phase as Eqs. (9), (20). The medical server makes a signature during the authentication phase as following Eq. (14). The adversary can't forge the signatures. So, our scheme can avoid the forgery attack.

\section{Integrity}

The patient's signature $\operatorname{Sig}_{P}$ can be verified by the doctor's public key as Eq. (7). Thus, the doctor can ensure the integrity of the message. And the doctor's signature $\operatorname{Sig}_{D}$ can be verified by the medical server's public key as Eq. (11). Thus, the medical server can ensure the integrity of the message. Also the medical server's signature $\operatorname{Sig}_{M S}$ can be verified by the doctor's public key as Eq. (17). Thus, the doctor can ensure the integrity of the message. And the doctor's signature $\mathrm{Sig}_{D 2}$ can be verified by the patient's public key as Eq. (23). Thus, the doctor can ensure the integrity of the message. Our scheme completes the integrity.

\section{E. Non-repudiation}

The party not only can check the validity through the signature, but also the party can't deny the message through the signature mechanism [11]. In our scheme, all parties make a signature as following Eqs. (5), (9), (14), (20). It is to say that our scheme has non-repudiation. In table 2 , we list the non-repudiation proof.

\section{Conclusions}

In this paper, we proposed a secure and efficient scheme for personal health care system. The doctor can easy make diagnoses and store the medical record. We think it is useful in our true life. Our scheme achieved the following security issues: (1) anonymity, (2) mutual authentication, (3) non-repudiation, (4) avoids forgery attack.

\begin{tabular}{|c|c|c|c|}
\hline Evidence & Evidence Issuer & Evidence Holder & Verification Equation \\
\hline $\operatorname{Sig}_{P}=S_{P R K}\left(M I D_{P} \| I D_{P}\right)$ & Patient & Doctor & $M I D_{P} \| I D_{P} \stackrel{?}{=} V_{P U K D}\left(\operatorname{Sig}_{P}\right)$ \\
\hline $\operatorname{Sig}_{D}=S_{P R K D}\left(I D_{D}\left\|M I D_{D}\right\| \operatorname{Cert}_{D}\right)$ & Doctor & Medical Server & $I D_{D}\left\|M I D_{D}\right\| \operatorname{Cert}_{D} \stackrel{?}{=} V_{P U K D}\left(\operatorname{Sig}_{D}\right)$ \\
\hline $\operatorname{Sig}_{M S}=S_{P R K M S}\left(I D_{M S}\left\|M I D_{M S}\right\| S K_{M S}\right)$ & Medical Server & Doctor & $I D_{M S}\left\|M I D_{M S}\right\| S K_{M S} \stackrel{?}{=} V_{P U K M S}\left(\operatorname{Sig}_{M S}\right)$ \\
\hline $\operatorname{Sig}_{D 2}=S_{P R K D}\left(I D_{D}\left\|M I D_{D}\right\| M_{D I N F}\right)$ & Doctor & Patient & $I D_{D}\left\|M I D_{D}\right\| M_{D I N F} \stackrel{?}{=} V_{P U K D}\left(\operatorname{Sig}_{D 2}\right)$ \\
\hline
\end{tabular}




\section{References}

[1] World Health Organization, "The top 10 causes of death," 2017.

[2] J. Wei, X. Hu, and W. Liu, "An improved authentication scheme for telecare medicine information systems," Journal of Medical systems, Vol. 36, 2012, pp. 3597-3604.

[3] Z. Zhu, "An efficient authentication scheme for telecare medicine information systems," Journal of Medical systems, Vol. 36, 2012, pp. 3833-3838.

[4] M. K. Khanand and S. Kumari, "An authentication scheme for secure access to healthcare services," Journal of Medical System, Vol. 37, No. 4, 2013, pp. 1-12.

[5] F. T. Bin Muhaya, "Cryptanalysis and security enhancement of Zhu's authentication scheme for Telecare medicine information system," Security and Communication Networks, Vol. 8, 2015, pp. 149-158.

[6] B. Yüksel, A. Küpçü and Ö. Özkasap, "Research issues for privacy and security of electronic health services," Future Generation Computer Systems, Vol. 68, 2017, pp. $1-13$.
[7] M. Hossain, S. R. Islam, F. Ali, K. S. Kwak, and R. Hasan, "An Internet of Things-based health prescription assistant and its security system design," Future Generation Computer Systems, 2017.

[8] A. K. Das, S. Zeadally and M. Wazid, "Lightweight authentication protocols for wearable devices," Computers \& Electrical Engineering, Vol. 63, 2017, pp. 196-208.

[9] F. Wu, X. Li, L. Xu, S. Kumari, M. Karuppiah, and J. Shen, "A lightweight and privacy-preserving mutual authentication scheme for wearable devices assisted by cloud server," Computers \& Electrical Engineering, Vol. 63, 2017, pp. 168-181

[10] C. T. Li, D. H. Shih and C. C. Wang, "Cloud-assisted mutual authentication and privacy preservation protocol for telecare medical information systems," Computer methods and programs in biomedicine, Vol. 157, 2018, pp. 191-203.

[11] P. Mohan and M. Singh, "Security Policies for Intelligent Health Care Environment," Procedia Computer Science, Vol. 92, 2016, pp. 161-167. 